\title{
Resection of the carina and oesophagus for malignant tumours of the oesophagus or tracheobronchial tree
}

\author{
TAKAAKI IKEDA, TADAAKI SAKAI, SHOJI SAKAI, SHIZUKA KASEDA, \\ RYOICHI OBITSU, MICHIO IVATSUKA \\ From the Department of Surgery, Tokyo Metropolitan Komagone Hospital, Tokyo, Japan
}

ABSTRACT Resection of the oesophagus together with the bifurcation of the trachea has been performed in three patients with oesophageal carcinoma or mucoepidermoid carcinoma of bronchial origin. Two patients had an uneventful recovery and survived five and 10 months but one patient died in the immediate postoperative period from aspiration pneumonia and respiratory failure. There has been no report of combined resection of the oesophagus and carina since Thompson's paper in 1973, but it appears to be indicated occasionally in patients with tracheobronchial or ocsophageal malignancy, particularly when it is associated with an oesophagobronchial fistula.

Carcinoma of the oesophagus extending to the tracheobronchial tree is usually regarded as a contraindication to surgical resection. Carinal resection and reconstruction can, however, now be performed for tumours of the trachea or main bronchi. ${ }^{1-6}$ If these are combined with oesophageal resection then healing of the tracheobronchial anastomosis may be compromised by interference with the blood supply to the anastomosis, as the bronchial vessels are removed with the oesophagus and the posterior membranous portion of the trachea is no longer supported by the oesophagus. In this paper we report three patients who had resection of the oesophagus and carina, two of whom survived.

\section{Case reports}

\section{CASE 1}

In July 1980 a 50 year old plasterer was admitted to our hospital because of haemoptysis of two months' duration. A chest radiograph showed narrowing of the lower trachea and bronchoscopy revealed a tumour of the left lower trachea, which biopsy showed to be a squamous carcinoma. A barium swallow showed stenosis of the oesophagus just below the carina and displacement of the upper oesophagus to the left (fig 1). Oesophagoscopy revealed an irregular ulcer posteriorly in the mid-

Address for reprint requests: Dr T Ikeda, Department of Surgery, Tokyo Metropolitan Komagone Hospital, Tokyo 113, Japan.

Accepted 8 November 1983 oesophagus and the patient was diagnosed as having tracheal invasion from lymph node metastases arising from an oesophageal carcinoma.

In view of increasing haemoptysis (up to $200 \mathrm{ml}$ a day) operation was performed through a right posterior thoracotomy. Ten rings of the lower trachea and one ring of the left main bronchus were resected together with the thoracic oesophagus. End to end anastomosis of the trachea to the right main bronchus and end to side anastomosis of the left main bronchus to the right main bronchus were performed (interrupted $2 / 0$ prolene sutures being used) according to the method of Barclay (fig 2). Proximal oesophagostomy and a Dedo's laryngeal release $^{7}$ were performed through a separate cervical incision. After operation the patient made an uneventful recovery and he did not require mechanical ventilation or aspiration of sputum. Five weeks later the alimentary tract was reconstructed by oesophagogastric anastomosis in the neck. A postoperative bronchoscopy showed that the main bronchi were patent and the anastomoses well healed without stenosis. The patient died five months after resection from local recurrence of the tumour.

\section{CASE 2}

A 58 year old man was admitted for investigation of chest pain and vomiting. Oesophagoscopy showed a malignant stenosis in the middle third of the oesophagus. The patient was about to receive preoperative irradiation but developed a bronchooesophageal fistula within one week of admission 


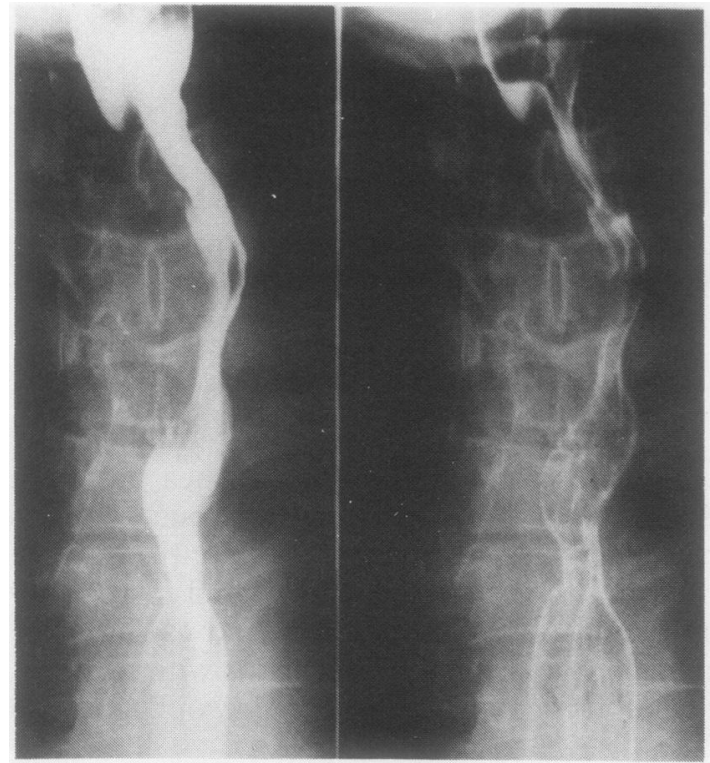

Fig 1 Case 1: Barium swallow showing a subcarinal stenosis and displacement of the upper oesophagus to the left. The trachea is outlined faintly as a result of aspiration.

and this caused a high fever that did not respond to treatment with various antibiotics. Fibreoptic bronchoscopy was then performed and showed a tumour involving the posterior membrane of the trachea and the origins of both main bronchi. In May 1981, two weeks after the occurrence of the fistula, operation was performed through a right posterolateral thoracotomy. Five rings of the lower trachea, four rings of the left main bronchus, the right upper lobe

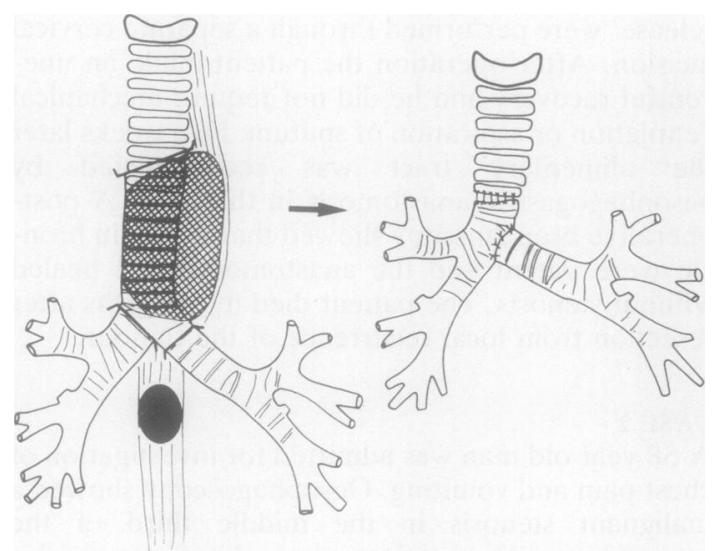

Fig 2 Case 1: Diagram showing the extent of carinal resection and method of reconstruction.

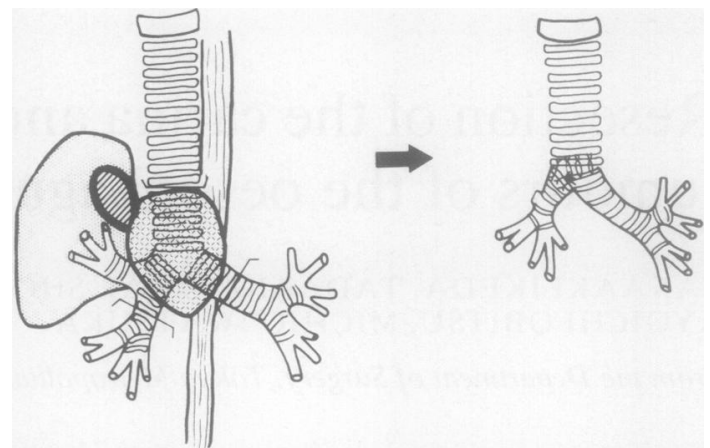

Fig 3 Case 2: Diagram showing the extent of carinal resection and the method of reconstruction.

(which was directly invaded by the oesophageal tumour and contained an abscess), and the right main bronchus were removed, together with the thoracic oesophagus. The trachea and left main bronchus were anastomosed end to end and the bronchus intermedius and the left main bronchus end to side, interrupted $2 / 0$ and $3 / 0$ prolene sutures being used (fig 3 ). The resected specimen showed the opening of the fistula just above the carina (fig 4). During operation bloody exudate was aspirated into the left lung through the fistula and respiratory support was necessary in the postoperative period. A chest radiograph taken the next day showed extensive infiltrate in the left lung and the patient died on the eight postoperative day from respiratory failure.

\section{CASE 3}

In May 1982 a 69 year old retired businessman was admitted to hospital with dysphagia of one month's

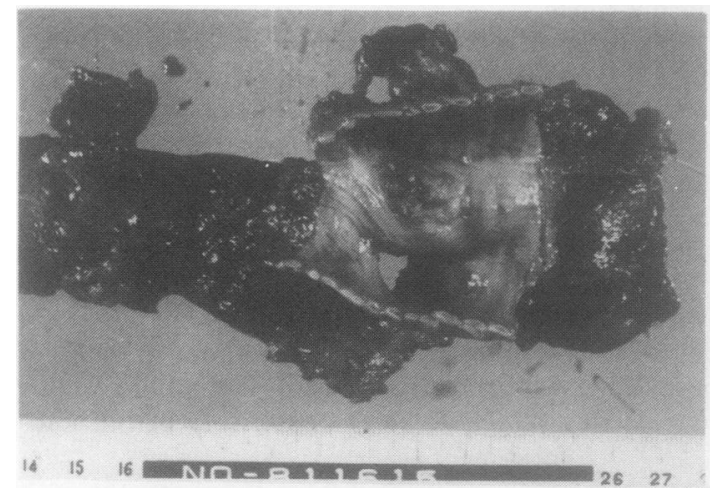

Fig 4 Case 2: The resected specimen showing the site of the oesophagobronchial fistula (above) and the opening of the left main bronchus (below). The scale is in centimetres. 


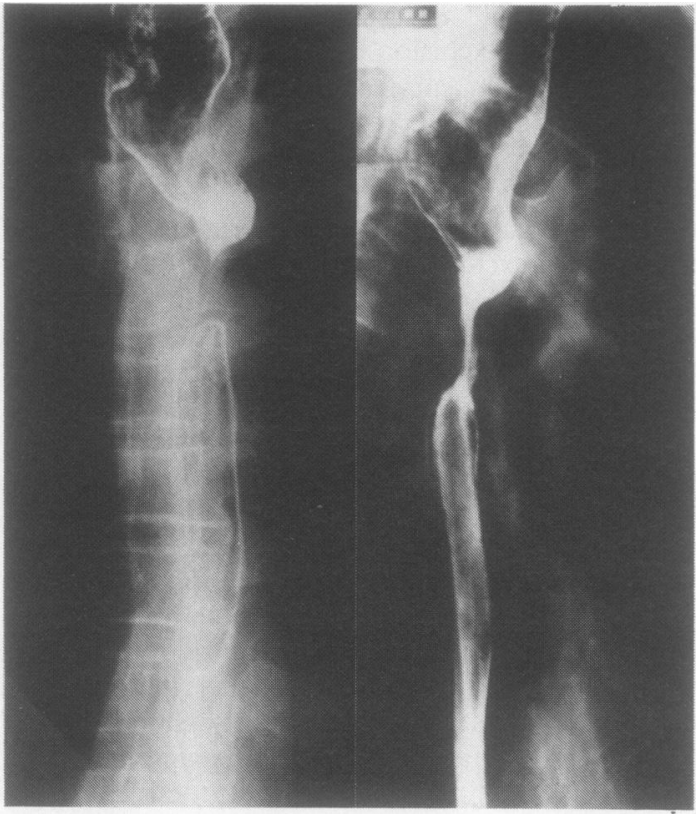

Fig 5 Case 3: Barium swallow showing stenosis of the mid-oesophagus.

duration. A barium swallow showed stenosis of the middle third of the oesophagus (fig 5) and fibreoptic bronchoscopy revealed malignant inva-

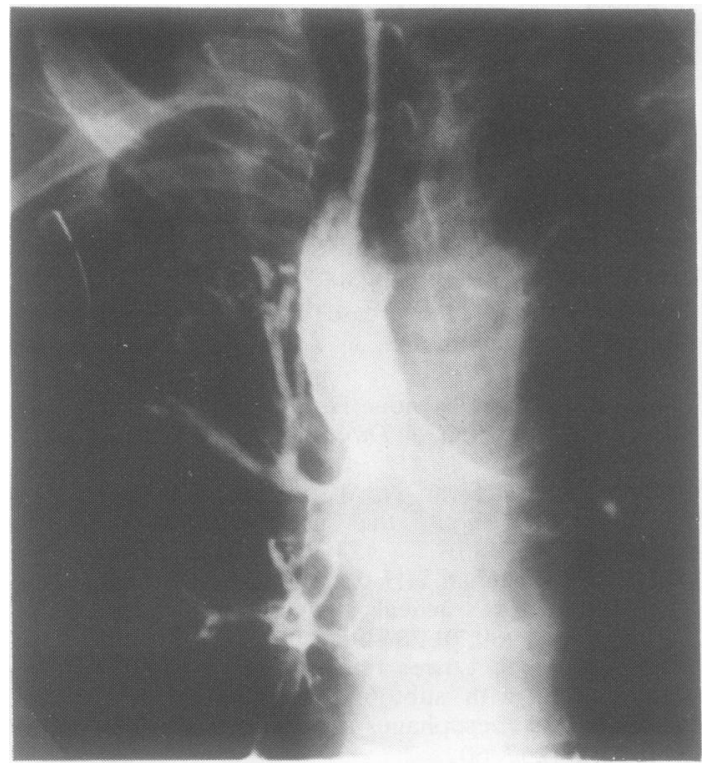

Fig 6 Case 3: Preoperative right sided bronchogram showing deformity of the carina and the tumour extending to the bronchus intermedius.
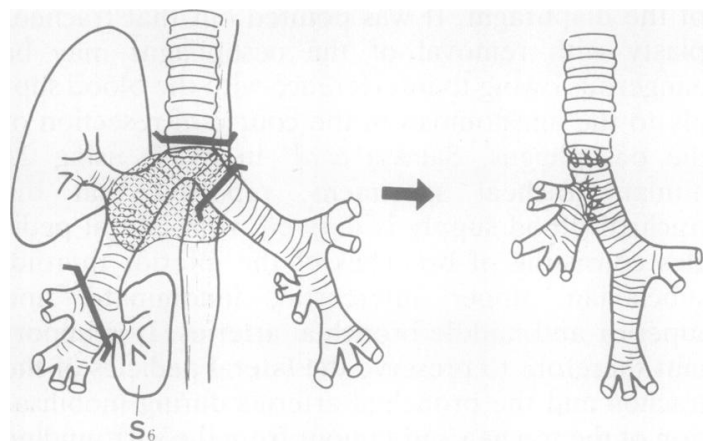

Fig 7 Case 3: Diagram showing the extent of carinal resection and the method of reconstruction.

sion of the posterior part of the lower trachea extending down to the orifice of the apical segmental bronchus of the right lower lobe and affecting the mouth of the right upper lobe bronchus. A bronchogram showed deformity of the carina and bronchus intermedius (fig 6). Biopsy specimens from the oesophagus did not show tumour but those from the bronchus showed epidermoid carcinoma. The primary tumour was thought to arise in the oesophagus and operation was performed through a right posterolateral thoracotomy with resection of the carina, the right upper lobe, the right mainstem bronchus, and the apical segment of the right lower lobe, together with the thoracic oesophagus. Reconstruction of the carina was by end to end anastomosis of the trachea and left main bronchus with end to side anastomosis of the bronchus intermedius to the left main bronchus, the anastomosis being wrapped with pedicled pleura (fig 7). Reconstruction of the alimentary tract was by oesophagogastrostomy through the retrosternal route. The patient made a good recovery from the operation and was discharged after 40 days. Pathological examination of the resected specimen showed a mucoepidermoid carcinoma of bronchial origin. Ten months later he died suddenly at home, possibly from a cardiac cause, though no necropsy was performed. Before his death there was no obvious evidence of recurrence.

\section{Discussion}

Resection and reconstruction of the carina for tumours of the trachea and bronchus with successful results have been reported by Grillo et al,' Mathey et al,$^{2}$ and others. ${ }^{3-6}$ In 1973 Thompson ${ }^{8}$ reported a case of oesophageal carcinoma that was treated by resection of the carina and thoracic oesophagus, the posterior carina being reconstructed from a portion 
of the diaphragm. It was pointed out that tracheoplasty with removal of the oesophagus may be dangerous owing to interference with the blood supply to the anastomosis in the course of resection of the oesophagus. Salassa et al in 1977, using 21 human tracheal specimens, reported that the tracheal blood supply is derived from lateral pedicles consisting of branches of the inferior thyroid, subclavian, upper intercostal, innominate, and superior and middle bronchial arteries. It is important therefore to preserve the lateral pedicles of the trachea and the bronchial arteries during mobilisation of the trachea and tumour from the surrounding tissues. After completion of the anastomosis a pedicled strip of pleura or pericardium can be wrapped around the suture line to protect against anastomotic dehiscence.

Anaesthetic technique is also important and the anaesthetist must understand precisely the nature of the lesion, the airway problem, and the surgery that is intended so that a mutually acceptable plan can be made for ventilation during the procedure. Usually we do not use a double lumen endotracheal tube because of obstruction at the level of the carina. Our second patient, however, died as a result of aspiration through a fistula and we now use a double lumen tube when a fistula is present. Once the bronchus is divided below the tumour then ventilation is performed through the left main bronchus and across the field of operation. If this does not produce sufficient oxygenation then an additional tube is inserted into the right lung, which is ventilated with pure oxygen. When the anastomoses are complete ventilation is re-established through the trachea.

With modern techniques up to half of the trachea can be safely resected with end to end anastomosis. In this series the greatest length resected was in patient 1 , who had 10 rings removed, combined with a laryngeal release to facilitate anastomosis. With regard to carinal reconstruction, end to end anastomosis of the right and left main bronchi to the trachea can be performed when the resected segment is short. ${ }^{2-57}$ If the resected segment is longer, an end to end anastomosis is performed between the trachea and the right main bronchus with an end to side anastomosis of the left main bronchus to the bronchus intermedius. ${ }^{6}$ In our third case the trachea and left main bronchus were anastomosed with an end to side anastomosis between the bronchus intermedius and the left main bronchus.

For patients with malignant oesophagobronchial fistula the prognosis is very poor, but survival can be prolonged if aspiration and reflux through the fistula can be prevented. Peroral oesophageal intubation is one method of palliation but yields a mean survival of only 2.5 months. ${ }^{10}$ Better palliation is obtained by retrosternal oesophagogastric bypass with oeosophageal exclusion. ${ }^{112}$ Orringer and Sloan"1 reported a mean survival of six months with this treatment, and Weaver and Matthews ${ }^{12}$ reported one patient with an irradiated oesophageal carcinoma and fistula who survived 22 months with a retrosternal oesophagogastric bypass and oesophageal exclusion. This is probably the method of choice for most patients with malignant oesophagobronchial fistula but it was not possible in patient 2 , who had a lung abscess resulting from direct perforation of his oesophageal carcinoma.

Lolley et al ${ }^{10}$ reported one patient with malignant oesophagobronchial fistula who was alive more than five years after pneumonectomy and oesophagectomy with reconstruction of the colon. Occasionally therefore there is a place for resection of tumours affecting both the oesophagus and the tracheobronchial tree. It is appropriate particularly for low risk young patients with relatively small tumours and requires a precise preoperative assessment of the extent of the tumour and careful planning of the operative procedure, which will include reconstruction of both the alimentary and the tracheobronchial tracts.

\section{References}

' Grillo HC. Bendixen HH, Gephart T. Resection of the carina and lower trachea. Ann Surg 1963;158: 889-93.

${ }^{2}$ Mathey J. Binet JP, Galey JJ, et al. Tracheal and tracheobronchial resections. Techniques and results in twenty cases. J Thorac Cardiovasc Surg 1966; 51:1-11.

${ }^{3}$ Eshapasse H. Les Tumeurs trachéales primitives traitement chirurgical. Rev Fr Mal Respir 1974; 2: $425-46$.

${ }^{4}$ Themar TE, Kerr JH, Nelems JM, et al. Carinal resection. A report of two cases and a description of the anesthetic technique. J Thorac Cardiovasc Surg 1976;71:314-20.

${ }^{5}$ Ishihara $\mathrm{T}$, Ikeda $\mathrm{T}$, Inoue $\mathrm{H}$, et al. Resection of cancer of lung of carina. J Thorac Cardiovasc Surg 1977; 73: $936-43$.

- Barclay RS, McSwan N, Welsh TM. Tracheal reconstruction without the use of grafts. Thorax 1957;12:177-80.

${ }^{7}$ Dedo HH, Fishman HH. Laryngeal release and sleeve resection for tracheal stenosis. Ann Otol Rhinol Laryngol 1969;78:285-96.

${ }^{8}$ Thompson DT. Lower tracheal and carinal resection associated with subtotal oesophagectomy for carcinoma of oesophagus involving trachea. Thorax 1973;28:257-60.

- Salassa JR, Pearson BW, Payne WS. Gross and microscopical blood supply of the trachea. Ann Thorac Surg 1977;24:100-7. 
${ }^{10}$ Lolley DM, Ray JF, Ransdell HT, et al. Management of malignant esophagorespiratory fistula. Ann Thorac Surg 1978;25:516-20.

$"$ Orringer MB, Sloan H. Substernal gastric by-pass of the excluded thoracic esophagus for palliation of

\section{Book notices}

Manual of Pulmonary Surgery. EW Humphrey, DL McKeown. (Pp 259; about £100.) Springer-Verlag. 1983.

This is the latest in a series of operative surgery atlases, which is designed to cover the whole range of surgical specialties with one volume for each subject. This volume deals with the surgery of the lungs, trachea, diaphragm, thymus, mediastinum, and chest wall. It is extensively illustrated with very good colour illustrations and the surgical techniques are generally up to date. There is rather more text than is customary in works of this type, but this increases the value of the volume for the trainee, as the general principles are well described in addition to the details of operative technique. It is probably the best single volume guide to non-oesophageal thoracic surgery currently available. While it is unlikely to be purchased by individual readers it should be regarded as an essential up to date reference book in all units concerned with this branch of surgery.-HRM

Thoracic Drainage. Noel H Fishman. (Pp 140; £14.40.) Year Book Medical Publishers. 1983.

Drainage of intrathoracic collections of air or fluid is a subject that is frequently misunderstood and mishandled. This paperback volume goes some way towards solving this problem but does not wholly succeed. It is intended as a practical manual on the drainage of the pleura and pericardium and is profusely illustrated with black and white diagrams. Much of the book is written in the first person singular and some of the statements would not meet with general agreement. There are also some omissions in that there is no clear account of the fundamental physical laws that govern evacuation of the pleura and no discussion of open versus closed drainage. Despite these criticisms the volume may be useful for junior hospital staff, physicians concerned occasionally in the management of these problems, and nurses, though it should not be regarded as the final and authoritative work on this subject. Essentially it is more suitable for being read at a single sitting than for repeated reference. At the price of $£ 14.40$ I would be inclined to borrow this small volume from the library.HRM esophageal carcinoma. J Thorac Cardiovasc Surg 1975;70:836-51.

12 Weaver RM, Matthews HR. Palliation and survival in malignant oesophago-respiratory fistula. $\mathrm{Br} J$ Surg 1980;67:539-42.

Intensive Care of the Surgical Cardiopulmonary Patient. 2nd ed. William E Neville. (Pp 412; £40.) Chicago: Year Book Medical Publishers Inc. 1983.

This multiple author book is intended for thoracic surgical residents, medical students, and nurses. The scope is wide, with chapters on the care of the adult and paediatric cardiac surgical patient, thoracic surgery (excluding the oesophagus), and, strangely, anaesthesia. The strength of the book lies in its clear descriptions of disordered physiology but it suffers from reduplication and the need to hunt through several chapters to obtain a guide to the management of a particular problem. Practical advice on treatment is patchy. Ionotropic drugs are fully discussed-twice. The management of renal failure is dismissed in a paragraph, and although the principle of the intra-aortic balloon pump is described there is nothing on the correct handling of the machine. Inevitably, the views of the authors conflict on occasion, but it is surprising to see towel clip traction being advocated for the treatment of flail chest in 1983, and pulmonary contusion discussed without mention of steroid or diuretic treatment. There is much to interest and instruct in this book, but it is unlikely to supplant the excellent British texts on the intensive care unit bookshelf.DBC 